\title{
Effect of silicon delta-doping density on optical properties of type-II InAs/GaAsSb quantum dots
}

\author{
Yeongho Kim ${ }^{\mathrm{a}, *}$, Keun-Yong Ban ${ }^{\mathrm{a}, 1}$, Darius Kuciauskas ${ }^{\mathrm{b}}$, Patricia C. Dippo ${ }^{\mathrm{b}}$, \\ Christiana B. Honsberg ${ }^{a}$ \\ ${ }^{a}$ Department of Electrical Engineering, Arizona State University, Tempe, AZ 85287-5706, USA \\ ${ }^{\mathrm{b}}$ National Renewable Energy Laboratory, Golden, Colorado 80401, USA
}

\begin{abstract}
We have investigated the optical properties of type-II InAs/GaAs ${ }_{0.83} \mathrm{Sb}_{0.17}$ quantum dots (QDs) with different silicon delta-doping densities of $5 \times 10^{10}, 5 \times 10^{11}$, and $2 \times 10^{12} \mathrm{~cm}^{-2}$ using photoluminescence (PL). The PL spectra of the QD ground state (GS) emission peaks for the samples are blueshifted at a slower rate with increasing the doping density due to the enhanced radiative recombination rate of the carriers. The PL intensity ratio of the GS emission to the first excited state emission increases with the doping density, which is indicative of the faster radiative recombination at the GS subbands with the doping density. The redshift rate of the GS emissions becomes faster at a high temperature $(>130 \mathrm{~K})$ as the doping density increases up to $5 \times 10^{11} \mathrm{~cm}^{-2}$ resulting from the quantum confined Stark effect by the electric field of the ionized dopants, and decreases at an increased doping density of $2 \times 10^{12} \mathrm{~cm}^{-2}$ due to the enhanced QD size uniformity. Time-resolved PL exhibits that the QD sample doped at $5 \times 10^{10} \mathrm{~cm}^{-2}$ has a longer total radiative lifetime than the undoped sample, and a further increase in the doping density to $2 \times 10^{12} \mathrm{~cm}^{-2}$ decreases the lifetime due to the enhancement of the radiative recombination through fast carrier relaxation.
\end{abstract}

\section{Keywords}

A1. Low dimensional structures

A3. Molecular beam epitaxy

B1. Antimonides

B2. Semiconducting III-V materials

\footnotetext{
${ }^{*}$ Corresponding author.

E-mail address: ykim172@asu.edu (Y. Kim).

${ }^{1}$ Present address: Applied Materials, Inc., Santa Clara, CA 95054-3299, USA
} 


\section{Introduction}

Self-assembled InAs/GaAs quantum dot (QD) systems have been extensively studied over the last decades for optoelectronic devices such as QD laser diodes [1], QD infrared photodetectors (QDIPs) [2], and intermediate band solar cells (IBSCs) [3-5] because of their strong carrier confinement and delta-function-like density of states.

In particular, InAs/GaAsSb QD systems have been recently suggested as a promising material candidate owing to their structural advantages over the InAs/GaAs QDs for improving the device performances of QDbased IBSCs. Unlike InAs/GaAs QDs with a large valence band offset (VBO), the VBO of InAs/GaAsSb QDs can be calibrated by varying Sb compositions [6]. The QD structures with a negligible VBO are predicted to have an improved open-circuit voltage $\left(\mathrm{V}_{\mathrm{oc}}\right)$ which is unobtainable from the InAs/GaAs QD based IBSCs [7]. Moreover, the carrier lifetime in the QDs can be prolonged by increasing Sb composition, resulting from the change in the energy band alignment from type-I to type-II structure [8]. Along with these advantages, as the intermediate band is half-filled with electrons, the short-circuit current density $\left(\mathrm{J}_{\mathrm{sc}}\right)$ of the IBSCs will be increased due to the absorption of the subband gap photon energies [4]. To do this, the silicon (Si) delta $(\delta)$ doping can be employed as an effective way to fill the electronic states of QDs with electrons because of its high electron-injection efficiency [9]. Despite such importance, the optical properties of $\mathrm{Si} \delta$-doped type-II InAs/GaAsSb QDs have not been sufficiently addressed [10-12].

In this work, we report on the optical properties of type-II InAs QDs embedded in GaAsSb layers with different Si $\delta$-doping densities. We find that the blueshift rate of the ground state (GS) emission under increasing photoexcitation becomes slower with increasing the doping density. The increase in the relative PL intensity of the GS emission to the first excited state (ES) emission with the doping density indicates that the radiative recombination rate at the GS subband levels is faster with increasing the doping density. In addition, the doping density affects the redshift rate of the GS emissions with increasing temperature. Further, timeresolved PL reveals that the total radiative lifetime of the lightly doped QD sample $\left(5 \times 10^{10} \mathrm{~cm}^{-2}\right)$ becomes longer than that of the undoped sample. When the doping density increases up to $2 \times 10^{12} \mathrm{~cm}^{-2}$ the radiative lifetime is shorter through the enhanced radiative recombination.

\section{Experiment}


All samples were grown by a solid-source molecular beam epitaxy (MBE) system. After the thermal deoxidation at $580^{\circ} \mathrm{C}$, a GaAs buffer layer of $400 \mathrm{~nm}$ was grown at $600^{\circ} \mathrm{C}$ on a semi-insulating GaAs (001) substrate and the substrate temperature was ramped down to $500^{\circ} \mathrm{C}$. At the lowered substrate temperature, a 10 nm-thick GaAsSb barrier with $\mathrm{Sb}$ to As flux ratio of 0.03 was grown and then an InAs layer with a nominal coverage of 2 monolayers (MLs) was grown at a growth rate of $0.04 \mathrm{ML} / \mathrm{s}$, followed by growing a $10 \mathrm{~nm}$-thick GaAsSb barrier. During the growth of the GaAsSb barrier, Si $\delta$-doping was performed $2 \mathrm{~nm}$ below the InAs QD layer with areal doping densities of $5 \times 10^{10}, 5 \times 10^{11}$, and $2 \times 10^{12} \mathrm{~cm}^{-2}$. The QD structures were finally capped with a $200 \mathrm{~nm}$-thick GaAs layer at $600^{\circ} \mathrm{C}$ for continuous-wave (cw) and time-resolved photoluminescence (TRPL) studies. As a reference sample, an undoped sample without $\mathrm{Si} \delta$-doping was also grown under the same growth conditions. The $\mathrm{Sb}$ composition of GaAsSb was determined to be $17 \%$ by double crystal $\omega-2 \theta \mathrm{XRD}$ measurements, and thus the as-grown samples in this study have type-II energy band alignments.

To examine the optical properties of the QD samples, we utilized a Nd:YAG laser $(\lambda=532 \mathrm{~nm})$ and a liquid nitrogen cooled InGaAs photodetector for cw-PL measurements, and a time-correlated single photon counting (TCSPC) system with a $200 \mathrm{fs}$ Ti:Sapphire laser $(\lambda=800 \mathrm{~nm})$ with a repetition rate of $250 \mathrm{kHz}$ to measure the decay transients of the QD emissions at 4.2 K for TRPL measurements. The penetration depth of the lasers for cw-PL and TRPL system exceeded the epilayer thickness of the QD samples and hence the carrier injection level by the optical pumping was expected to remain same for cw-PL and TRPL measurements in order to consistently characterize PL emission wavelength and carrier lifetime for the QD samples.

\section{Results and discussion}

Excitation power dependent PL measurements at $10 \mathrm{~K}$ were conducted to identify the band alignments of the InAs/GaAsSb QDs. The PL peak energies of the GS emissions of the all QD samples linearly increase with the cube root of the excitation power as shown in Fig. 1. The GS emission peaks of the samples A, B, C, and D experience the blueshifts of $28,35,17$, and $7 \mathrm{meV}$, respectively. The PL blueshift is observed in the quantum structures with a type-II band alignment due to the spatial separation of electrons and holes whereas it does not occur in type-I quantum structures [10]. The large blueshift of $35 \mathrm{meV}$ for the sample B originates from a strong band bending effect caused by the electron injection into the QDs. As more electrons are confined in QDs, the attractive Coulombic interactions between electrons confined in QDs and holes localized in GaAsSb layers 
increase, and thus strengthen the internal electric fields at the interface as seen in the inset of Fig. 1. The enhanced electric field causes more band bending, giving rise to a stronger blueshift in the PL spectrum.

On the other hand, the blueshift rate of the GS emission peak is slower with increasing the doping density up to $2 \times 10^{12} \mathrm{~cm}^{-2}$. This phenomenon can be explained as follows. The spatially separated carriers produce a triangular potential well with an electric field intensity of [13]

$$
F=\frac{2 \pi e n}{\varepsilon_{0}} \propto\left(\frac{P}{\gamma}\right)^{1 / 2}
$$

where $\mathrm{n}, \mathrm{P}$, and $\gamma$ are a photoexcited electron density in the GaAs and GaAsSb barrier layers, excitation power density, and radiative recombination rate, respectively. Further, The GS energy shift by the electric field is given by

$$
\Delta E_{C} \propto F^{2 / 3} \propto\left(\frac{P}{\gamma}\right)^{1 / 3}
$$

From the Eq. (2), we note that the GS energy shift is inversely proportional to the cube root of the radiative recombination rate $(\gamma)$. As shown in Fig. 1, the blueshift rate slows down as the doping level increases. This is attributed to the more electrons in the QDs injected from the $\delta$-doped plane since they induce the carrier-carrier scattering and, in turn, the fast carrier relaxation in the QD region. This results in the increased radiative recombination rate due to enhanced Coulombic interaction between built-in electrons and optically injected holes under the photoexcitation [14]. Since the doping level of the sample B is comparable with a QD number density of $\sim 5 \times 10^{10} \mathrm{~cm}^{-2}$, only one electron can preoccupy QD electronic levels [9]. Thus, the carrier-carrier scattering inducing the fast radiative recombination rate could be not pronounced to expedite the radiative recombination rate in the sample B.

Figure 2 exhibits the PL spectra separated by multiple Gaussian peaks at $10 \mathrm{~K}$ using an excitation power of $100 \mathrm{~mW}$ to observe the QD emissions from higher ES transitions. The GS PL peaks are centered at $1.103 \mathrm{eV}$ and $1.105 \mathrm{eV}$ corresponding to the $\mathrm{E}_{0} \mathrm{H}_{0}$ transitions for the samples $\mathrm{A}$ and $\mathrm{B}$, respectively. In addition, two $\mathrm{ES}$ peaks of $1.138 / 1.129 \mathrm{eV}$ and $1.158 / 1.157 \mathrm{eV}$ are observed corresponding to the $\mathrm{E}_{1} \mathrm{H}_{0}$ and $\mathrm{E}_{1} \mathrm{H}_{1}$ transitions for the samples A and B, respectively. These interband optical transitions are in a good agreement with our previous report [8]. The PL intensity ratio of $\mathrm{E}_{0} \mathrm{H}_{0}$ to $\mathrm{E}_{1} \mathrm{H}_{0}$ goes up with the doping density. This implies that the GS radiative recombination is enhanced by the Coulombic interaction across the InAs/GaAsSb interface as the doping level increases. Interestingly, while the increase of the PL intensity ratio is not significant for the sample B it turns out to be much pronounced in the case of the samples C and D. This trend is taken as evidence that the 
fast relaxation induced by the $\delta$-doping takes much more effect when the QD electronic levels are fully occupied. Compared to the samples A and B, the GS peak of the samples C and D is blueshifted by $\sim 33 \mathrm{meV}$. This trend is consistent with the report that the PL emission of GS transition for Si doped InAs/GaAsSb QDs and InAs/GaAs QDs was blueshifted by $\sim 80 \mathrm{meV}$ as compared to the undoped QD sample $[9,15]$. This large blueshift of the GS peak emission is likely due to the state filling effect in the QDs [15]. The nonparity transitions such as $\mathrm{E}_{1} \mathrm{H}_{0}$ and $\mathrm{E}_{1} \mathrm{H}_{2}$ where the selection rule is absent are observed due to the spatial separation of carriers in the type-II QD structure [8].

The temperature dependent behaviors of the GS peaks of the QD samples are displayed in Fig. 3. The GS peak energy decreases with increasing temperature due to the carrier redistribution in the QDs through the interdot hopping process from smaller QDs to larger ones with temperature [8] as seen in the inset of Fig. 3. In order to evaluate the redshift rate as a function of temperature, the PL peak energies are fitted using the empirical Varshni relation [16]. The redshift rates within the investigated temperature range from $10 \mathrm{~K}$ to $300 \mathrm{~K}$ are determined to be $0.30,0.36,0.48$, and $0.28 \mathrm{meV} / \mathrm{K}$ for the samples $\mathrm{A}, \mathrm{B}, \mathrm{C}$, and $\mathrm{D}$, respectively. The redshift rates of the InAs QDs are higher compared to that of $0.25 \mathrm{meV} / \mathrm{K}$ of an InAs bulk. The redshift rates of all QD samples are comparable in the low temperature region $(<130 \mathrm{~K})$ while the difference in the redshift rate becomes more prominent in the high temperature region $(>130 \mathrm{~K})$. The redshift rate is faster with the doping density up to $5 \times 10^{11} \mathrm{~cm}^{-2}$ and decreases at a doping density of $2 \times 10^{12} \mathrm{~cm}^{-2}$. As the temperature increases, more electrons in the $\delta$-doped plane are thermally activated and captured in the QDs, forming positively charged $\mathrm{Si}$ donors. The localized electric fields coming from the ionized donors are calculated to be 6.8, 67.2, and 268.8 $\mathrm{kV} / \mathrm{cm}$ for the samples B, C, and D, respectively [17]. These electric fields can redshift the interband transition energy $E$ which is known as quantum confined Stark effect following a quadratic dependence on the field $F$,

$$
E=E_{0}+\alpha F+\beta F^{2}
$$

where $\mathrm{E}_{0}, \alpha$, and $\beta$ are the transition energy at $\mathrm{F}=0$, permanent dipole moment, and polarization in the direction of the electric field, respectively. Accordingly, the redshift is expected to be more pronounced with increasing the doping density. The decrease in the redshift rate of the sample D, despite the highest doping density, is ascribed to the improved QD size uniformity as a result of the suppression of QD coalescence by the doping [19].

In order to study the effect of the doping density on the carrier lifetime in the QDs, TRPL measurements were carried out at $4.2 \mathrm{~K}$. It was known that the type-II InAs/GaAsSb QDs show the biexponential decay traces 
with a fast decay component and slow decay one [12]. The TRPL decay transients, as shown in Fig. 4, of the GS peaks are fitted by the biexponential decay function:

$$
I(t)=A_{1} \exp \left(-t / \tau_{1}\right)+A_{2} \exp \left(-t / \tau_{2}\right)
$$

where I is the integrated PL intensity, $\mathrm{A}_{1(2)}$ is the fitting constant, and $\tau_{1(2)}$ is the carrier lifetime. The short carrier lifetime $\tau_{1}$ results from the short-range radiative recombination of the carriers near the heterointerface at the initial decay stage. As the spatially separated carriers are more recombined, the band bending effect is reduced, weakening the wavefunction overlap of elections and holes. As a result, the reduction of recombination probability of the carriers gives rise to a long-range radiative lifetime $\tau_{2}$. As seen in Fig. 4 , the $\tau_{1}$ at the initial recombination stage becomes shorter with increasing the doping density. After the initial recombination, the $\tau_{2}$ increases with the doping density up to $5 \times 10^{10} \mathrm{~cm}^{-2}$. However, a further increase in the doping density results in the decrease of the $\tau_{2}$. This carrier lifetime dependence on the doping density is in accordance with the tendency showing that the radiative recombination rate increases with the doping density as stressed in Fig. 1.

A possible mechanism from the TRPL data taken here is as follow. The electrons from the $\delta$-doped plane preoccupy the QD electron subband levels. When the $\delta$-doping level of $5 \times 10^{10} \mathrm{~cm}^{-2}$ is used beneath the QD plane the QD electron GS is half-filled. Since the electrons and holes are spatially separated in the QD structure, the carrier-carrier scattering in the type-II structure may not occur as much as type-I structures when the $\delta$ doping is utilized as an electron supply. Therefore, the half-filled QD electron GS could be not enough to introduce a significant carrier-carrier scattering that results in the carrier relaxation and, in turn, electron-hole recombination in the QDs. As seen in Fig. 4, the sample B $\left(5 \times 10^{10} \mathrm{~cm}^{-2}\right)$ has a longer total radiative recombination time compared to the sample A (undoped). It is because the fast carrier relaxation by carriercarrier scattering in the QD region is not induced when the QD electronic levels are lightly filled. Nevertheless, when the doping level is increased up to $2 \times 10^{12} \mathrm{~cm}^{-2}$ the QD electronic levels are fully occupied. This causes the fast carrier relaxation in the QDs and enhanced radiative recombination [20] leading to the creation of a strong band bending across the interface between InAs/GaAsSb as explained in Fig. 1. When considering the application of the $\delta$-doped QDs to IBSCs, the long carrier lifetime in the QDs is needed to improve the photogenerated charge collection which is a key parameter affecting the efficiency of the QD-based IBSCs [21]. We note that the type-II QDs with optimized doping density have a potential for the realization of the highefficiency IBSCs owing to the increased carrier lifetime. 


\section{Conclusion}

In conclusion, we have studied the optical properties of Si $\delta$-doped type-II InAs/GaAsSb QDs. At the low temperature of $10 \mathrm{~K}$, the increased electron population in the QDs by the $\delta$-doping slows down the PL blueshift rate by the enhanced carrier-carrier scattering, and increases the PL intensity ratio of the GS emission to the first ES emission, indicating the faster radiative recombination at the GS subbands. Further, the redshift rate of the GS emissions with temperature ( $>130 \mathrm{~K}$ ) is accelerated with the doping density up to $5 \times 10^{11} \mathrm{~cm}^{-2}$ and drops down at a further increased doping density of $2 \times 10^{12} \mathrm{~cm}^{-2}$. From TRPL measurement, it is demonstrated that the total radiative lifetime is extended when the $\delta$-doping level of $5 \times 10^{10} \mathrm{~cm}^{-2}$ is used. However, when the $\delta$-doping density increases up to $2 \times 10^{12} \mathrm{~cm}^{-2}$ the total radiative lifetime decreases due to the enhancement of the radiative recombination induced by the fast carrier relaxation. The total radiative lifetime of the QDs is highly influenced by the QD electronic subband occupation which is controllable by calibrating the $\delta$-doping levels.

The trends of the optical properties of InAs/GaAsSb QDs observed at low temperatures will translate for IBSCs operating at higher temperatures. This is because the extent of the electron injection from the $\delta$-doped plane to the QD plane remains similar at high temperatures due to the high electron injection efficiency through the thermal excitation of electrons out of the $\delta$-doped plane and/or direct tunneling to the QD plane. Thus, the built-in electrons by the $\delta$-doping in QD electronic levels will induce the same doping effects at room temperature as they did at low temperatures.

Our findings suggest that the Si $\delta$-doping in type-II InAs/GaAsSb QDs is an effective method to fill the subband levels of the QDs with electrons and to tune both the interband carrier transitions and carrier lifetimes, which are the highly required features to improve the IBSC performances.

\section{Acknowledgments}

This material is based upon work primarily supported by the National Science Foundation (NSF) and the Department of Energy (DOE) under NSF CA No. EEC-1041895. Any opinions, findings and conclusions or recommendations expressed in this material are those of the authors and do not necessarily reflect those of NSF or DOE. This work was supported by the National Renewable Energy Laboratory as a part of the Non- 
Proprietary Partnering Program under Contract No. De-AC36-08-GO28308 with the U.S. Department of Energy.

\section{Reference}

[1] I. P. Marko, N. F. Massé, S. J. Sweeney, A. D. Andreev, A. R. Adams, N. Hatori, and M. Sugawara, Appl. Phys. Lett. 87 (2005) 211114.

[2] S.-F. Tang, S.-Y. Lin, and S.-C. Lee, Appl. Phys. Lett. 78 (2001) 2428.

[3] A. Luque and A. Marti, Phys. Rev. Lett. 78 (1997) 5014.

[4] A. Martí, E. Antolín, C. R. Stanley, C. D. Farmer, N. López, P. Díaz, E. Cánovas, P. G. Linares, and A. Luque, Phys. Rev. Lett. 97 (2006) 247701.

[5] F. K. Tutu, P. Lam, J. Wu, N. Miyashita, Y. Okada, K.-H. Lee, N. J. Ekins-Daukes, J. Wilson, and H. Liu, Appl. Phys. Lett. 102 (2013) 163907.

[6] H. Y. Liu, M. J. Steer, T. J. Badcock, D. J. Mowbray, M. S. Skolnick, P. Navaretti, K. M. Groom, M. Hopkinson, and R. A. Hogg, Appl. Phys. Lett. 86 (2005) 143108.

[7] A. Luque and A. Marti, Adv. Mater. 22 (2010) 160.

[8] K.-Y. Ban, D. Kuciauskas, S. P. Bremner, and C. B. Honsberg, J. Appl. Phys. 111 (2012) 104302.

[9] K.-Y. Ban, W.-K. Hong, S. P. Bremner, S. N. Dahal, H. McFelea, and C. B. Honsberg, J. Appl. Phys. 109 (2011) 014312.

[10] T. T. Chen, C. L. Cheng, Y. F. Chen, F. Y. Chang, H. H. Lin, C.-T. Wu, and C.-H. Chen, Phys. Rev. B 75 (2007) 033310.

[11] Y. D. Jang, T. J. Badcock, D. J. Mowbray, M. S. Skolnick, J. Park, D. Lee, H. Y. Liu, M. J. Steer, and M. Hopkinson, Appl. Phys. Lett. 92 (2008) 251905.

[12] Y.-A. Liao, W.-T. Hsu, M.-C. Lee, P.-C. Chiu, J.-I. Chyi, and W.-H. Chang, Phys. Status Solidi C 6 (2009) 1449.

[13] Y. S. Chiu, M. H. Ya, W. S. Su, and Y. F. Chen, J. Appl. Phys. 92 (2002) 5810.

[14] K. Gundogdu, K. C. Hall, T. F. Boggess, D. G. Deppe, and O. B. Shchekin, Appl. Phys. Lett. 85 (2004) 4570.

[15] J. Phillips, K. Kamath, X. Zhou, N. Chervela, and P. Bhattacharya, Appl. Phys. Lett. 71 (1997) 2079.

[16] Y. P. Varshni, Physica 34 (1967) 149.

[17] E. F. Schubert, J. Vac. Sci. Technol. A 8 (1990) 2980.

[18] P. W. Fry, I. E. Itskevich, D. J. Mowbray, M. S. Skolnick, J. J. Finley, J. A. Barker, E. P. O'Reilly, L. R. Wilson, I. A. Larkin, P. A. Maksym, M. Hopkinson, M. Al-Khafaji, J. P. R. David, A. G. Cullis, G. Hill, and J. C. Clark, Phys. Rev. Lett. 84 (2000) 733.

[19] R. Duan, B. Wang, Z. Zhu and Y. Zeng, Jpn. J. Appl. Phys. 42 (2003) 6314.

[20] J. Siegert, S. Marcinkevičius, and Q. X. Zhao, Phys. Rev. Lett. 72 (2005) 085316. 
[21] A. Martí, N. López, E. Antolín, E. Cánovas, A. Luque, C. R. Stanley, C. D. Farmer, and P. Díaz, Appl. Phys. Lett. 90 (2007) 233510.

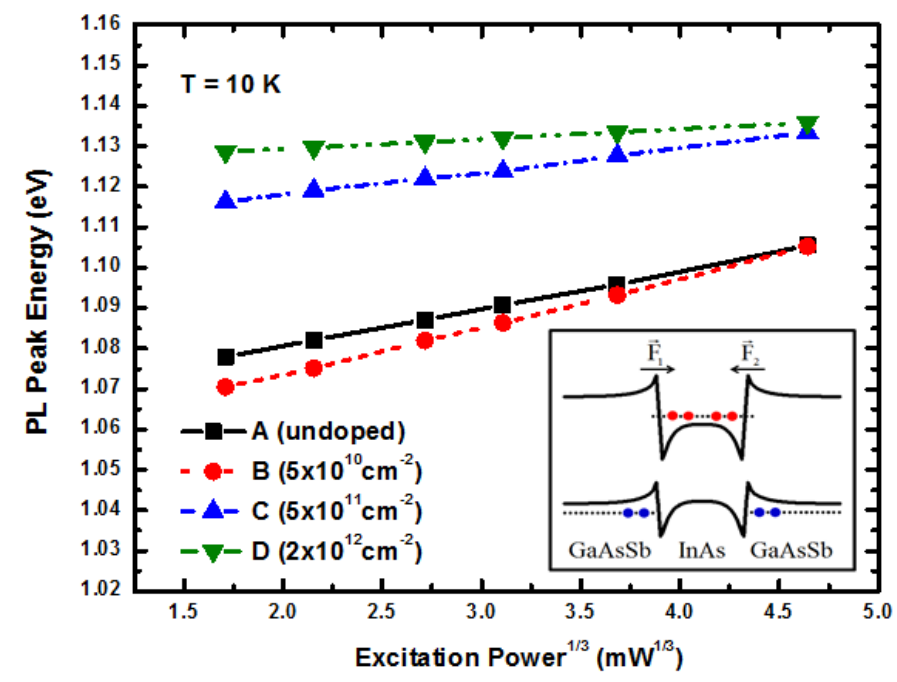

Fig. 1. GS PL peak energy shift against the cube root of the excitation power for the QD samples at $10 \mathrm{~K}$. The lines are leastsquares fits for the experimental data points. The inset shows a schematic band structure where the internal electric fields $F_{1(2)}$ are induced by the band bending effect. 


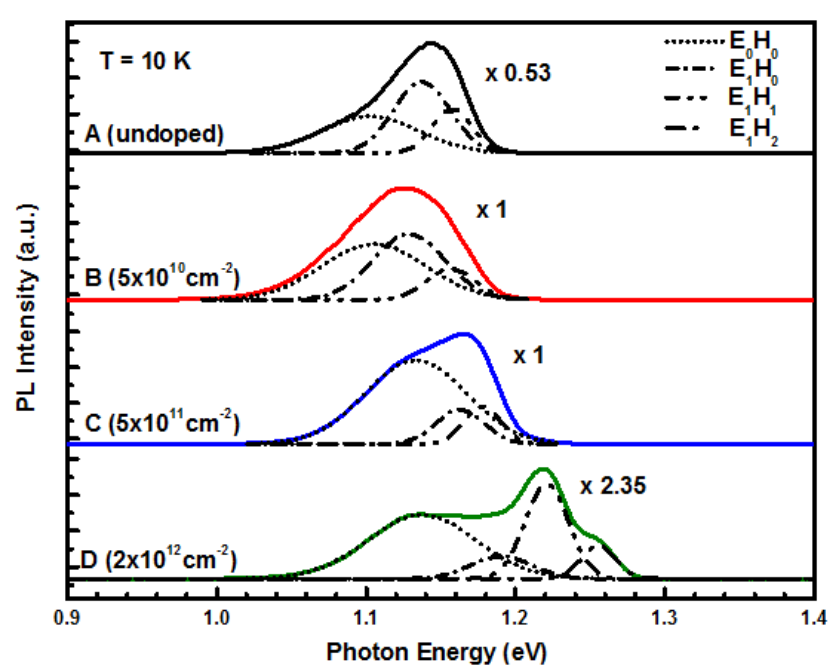

Fig. 2. PL spectra at $10 \mathrm{~K}$ at an excitation power of $100 \mathrm{~mW}$. Each PL spectrum was decomposed into Gaussian functions showing interband transitions. ( $\mathrm{E}_{0}, \mathrm{E}_{1}$ : electron ground and first excited state / $\mathrm{H}_{0}, \mathrm{H}_{1}, \mathrm{H}_{2}$ : hole ground, first, and second excited state).

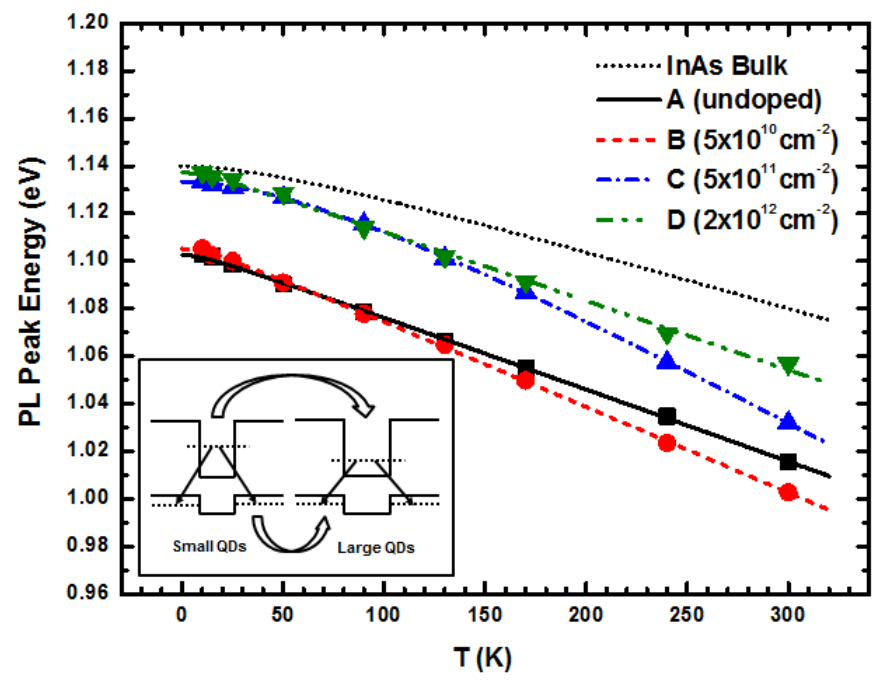

Fig. 3. Temperature dependence of the GS peak energies of all QD samples. The data points are fitted using the Varshni law. The inset displays the interdot hopping process with increasing temperature. 


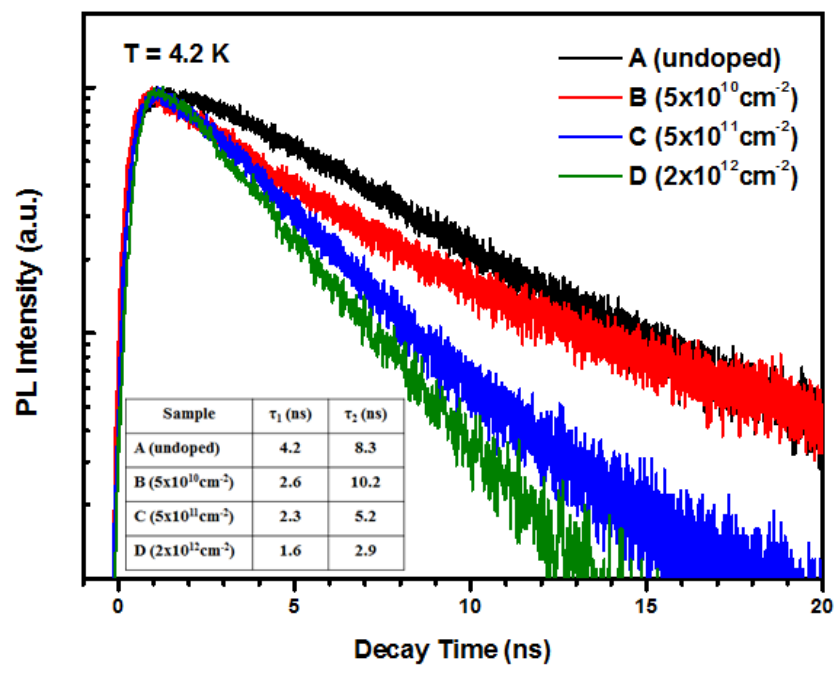

Fig. 4. TRPL decay transients of the GS peaks for the InAs/ $\mathrm{GaAs}_{0.83} \mathrm{Sb}_{0.17}$ QDs at $4.2 \mathrm{~K}$. The inset includes the fitted carrier lifetimes of $\tau_{1}$ and $\tau_{2}$. 